\title{
MACROSCOPY
}

\section{Vascular Pharmacology: Opportunities for Intervention}

\section{J Keiser}

The vascular wall, composed of adventitia, smooth muscle cells, and an endothelium, is no longer viewed as a static structure but rather as a highly mutable organ responsive to the local mechanical, hemodynamic, and neurohumoral milieus. The vasculature, along with the heart, distributes nutrient blood flow throughout the body. Resistance in the microvasculature is critical to regulation of arterial blood pressure; failure to appropriately modulate arterial tone leads to hypertension (HT). Disruption of the endothelium lining the vasculature induces luminal thrombosis. Inflammatory processes in the vessel wall are the early harbingers of atherosclerosis. This brief review looks at the major drug classes that have directly targeted vascular wall processes to treat HT, thrombosis, and atherosclerosis, with a focus on the future opportunities for research.

\section{Antihypertensives}

It is widely recognized that treating HT reduces the risk of stroke, coronary artery disease, heart failure, renal disease, and cardiovascular mortality. Several major classes of drugs are available to treat HT, including $\alpha$-blockers, $\beta$-blockers, vasodilators, calcium channel blockers, inhibitors of the renin-angiotensin-aldosterone (RAA) axis, and diuretics. Diuretics, introduced in the 1950s, were among the earliest classes of antihypertensives. Hydralazine, one of the earliest vasodilators, was also introduced in this era. Diuretics do not act directly on the vasculature but rather reduce extracellular volume. Follow-ons to diuretics were drugs that more specifically targeted the vessel wall. Numerous $\alpha$ - and $\beta$-blockers with varying degrees of receptor subtype selectivity altered the neurohumoral milieu in the vessel wall. Calcium channel blockers, another class of antihypertensives, act directly at the vascular smooth muscle cells to vasodilate. Blockade of the endogenous RAA pathway is the mechanism underlying several successful antihypertensive agents. There are drug classes that target different points in the RAA pathway, but these agents provide similar therapeutic benefit regardless of mechanism. The most recent and least tested of these RAA drugs are renin inhibitors that block angiotensin generation by directly inhibiting the ratelimiting enzyme in this pathway. Whether aliskiren, approved in the United States as an antihypertensive in 2007, provides any unique benefit compared with other RAA pathway drugs will be apparent only with further clinical experience.

The discovery and development of novel antihypertensives have been almost completely abandoned by the major pharmaceutical companies, with the exception of Merck, which entered a major collaboration with $\mathrm{NicO}$ to develop nitric oxide pathway-based agents. Although several vasoactive pathways have been explored preclinically as potential approaches to HT treatment, the challenge of introducing a new agent in a field that is arguably well satisfied is daunting. Most major classes of antihypertensives have gone generic, raising the bar for new agents to deliver increased value to the payer as well as the patient. Although this is not required for regulatory approval, many companies consider matching the morbidity and mortality (M\&M) data from currently approved agents to be important for the commercial success of new agents. The cost of conducting M\&M trials requires a large up-front investment that is not easily recouped.

Nonetheless, from a mechanistic perspective, there are two areas that could provide unique HT therapies or provide benefit in specific patient populations. Therapies that specifically target the endothelial cells lining the vasculature and contribute to regulation of tone are largely unexplored. PDE5 inhibitors, used to treat erectile dysfunction, are an example of drugs that improve endothelial function. PDE5 inhibitors have demonstrated antihypertensive efficacy in small phase II trials. PDE5 inhibition is but one of several ways to target the endothelium via nitric oxide pathways, endotheliumderived hyperpolarizing factors, or various prostanoid agents.

Another approach to modulating vascular tone involves modifying the extracellular matrix that provides the viscoelastic properties of the vasculature. It has been postulated that isolated systolic HT in the elderly may be the result of changes in the quantity and type of collagen in the vessel wall. The formation of advanced glycosylation end products (AGEs) leading to collagen crosslinking has been 
hypothesized to contribute to HT and vascular disease in diabetics. Although numerous approaches, including matrix metalloproteinase inhibitors, AGE inhibitors, and AGE breakers, have been profiled preclinically, none has demonstrated clinical proof of concept as a antihypertensive. As we gain a better understanding of the microstructure of the vasculature, including a more complete view of the dynamic nature of extracellular matrix and cell matrix interactions, new insights that could lead to new therapies await us. We have only begun to understand the high degree of plasticity in the vessel wall.

Interestingly, $\mathrm{HT}$ is a recognized side effect of more than one class of therapeutics. For example, the growth factor kinase inhibitors used to treat cancers are uniformly associated with HT. The use of recombinant erythropoietin in chronic renal failure patients induces HT in more than $25 \%$. A better understanding of the mechanisms responsible for elevating blood pressure with some of these agents may lead to novel strategies for treating HT. The status quo is that most hypertensive patients take two or three antihypertensive medications. The holy grail in this field remains a single agent that normalizes blood pressure regardless of the patient cohort.

\section{Antiplatelet agents}

It is the delicate balance between procoagulant and anticoagulant activities that ensures the fluidity essential to normal blood flow. Damage or disruption of the vascular endothelium exposes circulating platelets to subendothelium, triggering aggregation and formation of a hemostatic plug. As part of normal physiology, this stimulation of plasma coagulation factors allows generation of a fibrin clot, which reinforces the platelet plug. In pathological situations, clot formation occludes the blood vessel, resulting in ischemia.

Aspirin is the most widely recognized and used antiplatelet agent. By covalently acetylating cyclooxygenase, aspirin effectively blocks production of thromboxane $\mathrm{A}_{2}$ throughout the life of the exposed platelet. Platelets contain two $\mathrm{P} 2 \mathrm{Y}$ receptors, $\mathrm{P} 2 \mathrm{Y} 1$ and $\mathrm{P} 2 \mathrm{Y} 2$; targeting either or both can prevent platelet aggregation. Ticlopidine and clopidogrel are two examples of P2Y receptor antagonists with demonstrated clinical efficacy. Another antiplatelet mechanism works by blocking the platelet surface integrin receptor. The resultant drug class, glycoprotein IIb/IIIa inhibitors exemplified by abciximab, has shown benefit in conjunction with percutaneous angioplasty. However, the high cost, parenteral route of administration, short half-life, and bleeding risk limit use of IIb/IIIa inhibitors to interventional procedures.

The greatest opportunity in the field of antithrombotics is development of an orally active agent with reduced bleeding risk compared with state-of-the art interventions. Rather than targeting the platelet-vessel wall interaction, new agents under investigation seek to disrupt key zymogens in the coagulation cascade, such as factor Xa. Several factor Xa inhibitors are at various stages of clinical testing. Another critical component of coagulation is tissue factor (TF), expressed in the subendothelium. TF amplifies the coagulation cascade roughly 30,000-fold and thus exponentially drives clot formation. An orally active TF inhibitor has long been sought as a potential therapeutic. In theory, a TF inhibitor could provide anticoagulation with reduced bleeding risk compared with other inhibitors of the coagulation cascade. Thus far, no one has successfully brought an orally active TF inhibitor into clinical development.

Genetics has framed our understanding of thrombosis for centuries, as with, for example, the recognition of heritance patterns in hemophilia. Today we have the know-how to track several gene variations that impact hemostasis. More important, genotyping is now used to predict the effectiveness of agents such as coumadin and clopidogrel. The growth of personalized medicine will continue to provide physicians with the tools to match the drug to the patient.

\section{Antiatherosclerotics}

Despite several decades of targeted intervention, coronary heart disease (CHD, atherosclerosis), cerebrovascular disease, and peripheral vascular disease account for the majority of M\&M in the elderly in the United States. Risk factors for CHD include elevated low-density lipopro- tein cholesterol (LDL-C), reduced highdensity lipoprotein cholesterol (HDL-C), cigarette smoking, hypertension, and diabetes. Hypercholesterolemia is one means of disrupting the intact vascular endothelial barrier. Inflammatory macrophages and foam cells in the vessel wall are the early markers of atherosclerotic plaque formation. Atherosclerotic vascular disease progresses over many decades, but as these lesions grow, the likelihood of plaque rupture increases. Rupturing of an atherosclerotic plaque exposes circulating blood to subendothelial factors, triggering clot formation.

Recognition of hypercholesterolemia and the subsequent treatment of dyslipidemias have reduced CHD mortality by as much as $30-40 \%$. Lifestyle changes in diet and exercise are the first and critical step in any treatment of lipid disorders. The most effective and besttolerated dyslipidemia drugs are the 3-hydroxy-3-methylglutaryl-coenzyme A reductase inhibitors, or statins, which lower LDL-C. Other drugs for treating dyslipidemias include cholesterolabsorption inhibitors, bile acid resins, nicotinic acid, and fibric acid derivatives. Both statins and bile acid resins target LDL-C. Resins are used less frequently, primarily for statin-intolerant patients, but are less efficacious. Nicotinic acid has favorable effects on HDL-C and triglycerides in addition to LDL-C. Sustained-release formulations of niacin, which have successfully addressed the flushing liability, are important players in the treatment of low HDL-C. Fibric acid derivatives have a more variable therapeutic profile that is dependent on the patient population.

The combination of a cholesterolabsorption inhibitor and a statin is highly effective at lowering LDL-C. Interestingly, a large-scale M\&M trial of ezetimibe and simvastatin failed to decrease CHD events as compared with equivalent LDL-C lowering with a statin alone. The results from the Ezetimibe and Simvastatin in Hypercholesterolemia Enhances Atherosclerosis Regression (ENHANCE) trial have confounded researchers. In addition to new statins and statin combinations, other LDLC-lowering agents explored over the 
past decade include microsomal triglyceride transfer protein inhibitors and acyl-coenzyme A cholesteryl acyltransferase inhibitors. The latter have largely been abandoned. Studies continue with microsomal triglyceride transfer protein inhibition, although at a slower pace and with great care, given the potential risk of hepatic steatosis.

In an effort to raise HDL-C, several companies have targeted cholesteryl ester transfer protein (CETP). In late 2006, phase III clinical trials with torcetrapib, the most advanced CETP inhibitor, were halted because of an imbalance in all-cause mortality. The cause of the increased risk with torcetrapib remains undetermined. Clinical trials with other CETP inhibitors have resumed, albeit cautiously. With the failure of torcetrapib, it is clear that any novel mechanism of HDL-C elevation will need to demonstrate $M \& M$ benefit. HDL-C elevation remains a tremendous opportunity for new therapeutics, but the cost of entry will be high. Continued scientific investment in the fundamental biology of HDL is critical.

Although treatment of dyslipidemias has had an enormous impact on overall cardiovascular M\&M, not every patient who meets current National Cholesterol Education Program guidelines for lipid management remains disease-free. For many years scientists have studied drug targets that directly alter atherosclerotic disease progression in the vessel wall. From agents to prevent vascular inflammation to agents that modify vascular trafficking of adhesion molecules, the opportunity is vast. Selectins, integrins, $\mathrm{T}$ cells, inflammatory monocytes, matrix metalloproteinases, and a host of cytokines have been implicated in the evolution of an atherosclerotic plaque. The field is hampered by the lack of animal models that recapitulate the complexity of human atherosclerotic disease and by our inability to fully elucidate early changes in the vascular wall in humans. Advances in the field of genomics should help us better understand and interrogate pathways involved in atherosclerosis.

At present, fewer than 2\% of the 671 clinical trials registered at http://www. ClinicalTrials.gov under the category of atherosclerosis represent novel drug entities. Forty percent of that extremely small subset target either soluble or lipoprotein PLA2; other mechanisms include chemokine receptor 2 and rho kinase.

Future breakthroughs in the treatment of atherosclerosis are critically dependent on better methodologies to image and temporally track changes in human vasculature over months and years as disease develops. Approval of a direct-acting antiatherosclerotic agent will require M\&M trials, but companies currently lack an intermediate step, or proof-of-concept measure, to reduce the risk of the larger M\&M investment.

\section{CONFLICT OF INTEREST}

The author declared no conflict of interest.

() 2009 ASCPT 